\title{
Effect of Heart Rate Increase on Dorsal Aortic Flow in the Stage 24 Chick Embryo
}

\author{
ANN DUNNIGAN, NORMAN HU, D. WOODROW BENSON, JR., AND EDWARD B. CLARK \\ Division of Pediatric Cardiology, Department of Pediatrics, The Johns Hopkins University School of Medicine, \\ Baltimore, Maryland 21205 [N.H., E.B.C.] and the Department of Cardiology, Children's Memorial Hospital, \\ Northwestern University Medical School, Chicago, Illinois 60614 [A.D., D.W.B.]
}

\begin{abstract}
We evaluated the effect of increased heart rate on cardiac output and stroke volume in the stage 24 chick embryo (day 4 of a 21-day incubation). Bloud flow was measured with a $20 \mathrm{MHz}$ pulsed-Doppler flowmeter. Heart rate was increased by pacing with square wave stimuli (1 ms duration, $<4 \mathrm{~mA}$ ). The sinus venosus was paced from bipolar Teflon-coated silver electrodes in eight embryos and the ventricular apex was paced in three embryos. The pacing rates were at the intrinsic heart rate (P:I); 125\% of intrinsic heart rate (P:125\%I); and $150 \%$ of intrinsic heart rate (P:150\%I). Physiologic measurements during pacing were compared to those obtained at the control intrinsic rate (I). We also evaluated the velocity profile of atrioventricular inflow and conotruncal outflow at intrinsic rate and during sinus venosus and ventricular pacing. With sinus venosus pacing, mean dorsal aortic blood flow was similar at control $\left(1.07 \pm 0.05 \mathrm{~mm}^{3} / \mathrm{s}\right)$ and P:I $\left(1.06 \pm 0.06 \mathrm{~mm}^{3} / \mathrm{s}\right)(\overline{\mathrm{x}} \pm \mathrm{SEM})$. However, at P:125\%I and $P: 150 \% I$, mean dorsal aortic blood flow decreased significantly (P:125\%I, $0.88 \pm 0.05 \mathrm{~mm}^{3} / \mathrm{s} ; \mathrm{P}: 150 \% \mathrm{I}, 0.67$ $\left.\pm 0.07 \mathrm{~mm}^{3} / \mathrm{s}\right)(p<0.05)$. Stroke volume per beat also decreased with increasing heart rates $\left(I, 0.41 \pm 0.02 \mathrm{~mm}^{3}\right.$; P:I, 0.39 $\pm 0.02 \mathrm{~mm}^{3} ;$ P:125\%I, 0.28 $\pm 0.02 \mathrm{~mm}^{3}$; P:150\%I, 0.18 $\left.\pm 0.02 \mathrm{~mm}^{3}\right)(p<0.05)$. With rapid sinus venosus pacing, the atrioventricular blood flow velocity profile showed a rate-dependent decrease in passive ventricular filling while active filling remained the same or increased slightly. Thus, rate-dependent passive ventricular filling may be one reason for relatively slow heart rates during early embryonic development. During ventricular pacing at the intrinsic heart rate, mean dorsal aortic blood flow decreased to near zero presumably secondary to loss of normal atrioventricular synchrony. We speculate that atrial or ventricular tachycardia would be lethal to an embryo. (Pediatr Res 22: 442-444, 1987)
\end{abstract}

The heart begins to function early in embryonic development. In the chick embryo, heart rate is $90 \mathrm{bpm}$ at the onset of blood flow and gradually increases during development to $210 \mathrm{bpm}$ at hatching.

The mechanisms that control heart rate in the embryo are not

Received January 26, 1987; accepted May 13, 1987.

Supported by NIH HL-14388 and funds from the Belfer Laboratory, The Helen B. Taussig Endowment, and the Walden W. and Jean Young Shaw Foundation. A.D. is the recipient of a Basil O'Connor Starter Research Grant from the March of Dimes. E.B.C. is the recipient of NIH RCDA HD-00376.

Correspondence and reprints Edward B. Clark, M.D., Division of Pediatric Cardiology, Box 631, University of Rochester School of Medicine, 601 Elmwood Avenue, Rochester, NY 14642 well defined. At the early stages, the sinus node and specialized conduction system are not histologically identifiable nor is the heart innervated by the autonomic nervous system. Yet, a chick embryo's heart rate responds to environmental factors. The heart rate slows with hypothermia (1) and increases with hyperthermia (2).

We hypothesized that heart rate and cardiac output are directly related to the preinnervated embryonic circulation. However, we found that a paced increase in heart rate decreased cardiac output and stroke volume by compromising the passive phase of ventricular filling.

\section{METHODS}

Fertile white Leghorn chick eggs were incubated to Hamburger-Hamilton stage 24 (3). The shell and its membranes were opened to expose the embryo. Blood flow velocity was measured with a $20 \mathrm{MHz}$ pulsed-Doppler meter from a 750 piezoelectric crystal positioned over the dorsal aorta, conotruncus or ventricular apex (4). Mean dorsal aortic blood flow was computed as the product of mean velocity and aortic area. Heart rate was measured from five phasic velocity peaks compared to time lines. Stroke volume per beat was calculated as mean dorsal aortic blood flow/heart rate. Conotruncal and atrioventricular blood flow velocities were evaluated qualitatively.

The embryonic heart was paced with square wave stimuli of 1 ms duration at twice diastolic threshold $(<4 \mathrm{~mA})$. Bipolar Tefloncoated silver electrodes ( $12 \mu$ diameter) were positioned on the right sinus venosus in eight embryos and on the ventricular apex in three embryos. Pacing the sinus venosus evaluated the effect of increased heart rate, presumably, without altering the sequence of cardiac excitation and contraction. Pacing the ventricle evaluated the effect of altering the cardiac excitation contraction sequence and loss of atrioventricular synchrony.

Mean dorsal aortic blood flow was measured sequentially: prior to pacing (control); pacing at the intrinsic heart rate (P:I), pacing at $125 \%$ of intrinsic heart rate $(\mathrm{P}: 125 \% \mathrm{I})$; pacing at intrinsic heart rate; pacing at $150 \%$ of intrinsic heart rate (P:150\%I); pacing at intrinsic heart rate; after the pacing protocol was completed $\left(\mathrm{I}^{\prime}\right)$. In this way, the effect of pacing alone could be evaluated as well as the effect of heart rate increase. A complete study was performed within $2 \mathrm{~min}$.

In separate experiments, pulsed-Doppler patterns of simultaneous atrioventricular inflow and conotruncal outflow blood flow velocities were evaluated at control and while pacing of the sinus venosus or ventricle.

We were unable to measure dorsal aortic pressure during pacing because of interference of the stimulus with the servonull pressure system.

The results are reported as mean \pm SEM and were tested for statistical significance at the $p<0.05$ level by analysis of variance and Tukey's HSD test. 


\section{RESULTS}

Sinus venosus pacing. Details of heart rate, the independent variable, are listed in Table 1. The intrinsic heart rates before and after the pacing protocols were similar. The initial paced rate averaged $6 \mathrm{bpm}$ greater than the intrinsic heart rate. Mean dorsal aortic blood flow was similar at baseline, with pacing near the intrinsic rate, and following the pacing protocol (Fig. 1). Mean dorsal aortic blood flow decreased $14 \%$ with pacing at $125 \%$ of intrinsic heart rate and $35 \%$ with pacing at $150 \%$ of intrinsic heart rate $(p<0.05)$. Stroke volume per beat decreased significantly with pacing at both 125 and $150 \%$ of intrinsic heart rate $(p<0.05)$ (Fig. 2).

Ventricular inflow velocity patterns did not change during sinus venosus pacing at the intrinsic heart rate. However, at more rapid pacing rates, the passive filling phase decreased as a consequence of shortened diastolic time (Fig. 3). Atrioventricular regurgitation was not identified.

Ventricular pacing. Pacing the ventricular apex at the intrinsic heart rate or faster caused a precipitous decrease in dorsal aortic blood flow without apparent atrioventricular insufficiency (Fig. 4). We noted an obvious loss of the active phase of filling with ventricular pacing.

\section{DISCUSSION}

The heart is the first functioning organ in the embryo. In the chick, contractions of the cardiac tube begin at stage $10+(36 \mathrm{~h})$ and blood flow commences shortly thereafter at stage $12(42 \mathrm{~h})$. Blood is propelled through the heart by rhythmic contraction and the endocardial and conotruncal cushions act like valves to prevent retrograde blood flow.

Depolarization of the heart tube begins in the right sinus venosus and sequentially passes to the atria, ventricle, and conotruncus (5). At these stages, there is no recognizable conduction system and the heart is not innervated. The pacemaker cells cannot be histologically identified but presumably are the progenitors of the sinus node.

In the chick embryo, the heart rate gradually increases with development. At the onset of blood circulation, the rate is 90 bpm and increases to $210 \mathrm{bpm}$ just prior to hatching. The reason for the increase in heart rate during ontogeny is unknown. The mechanism may relate to cell membrane characteristics that change during embryonic development (6).

Variation in the heart rate is a mechanism for regulating cardiac output in the chick as the environmental temperature affects heart rate and hemodynamic function. Hypothermia decreases heart rate, cardiac output, and increases vascular resistance without affecting stroke volume (1). Hyperthermia increases heart rate, cardiac output, and decreases vascular resistance again without affecting stroke volume (2). Yet, it is unclear whether these responses represent an isolated effect of heart rate or a more integrated response of the cardiovascular system.

Our current study was designed to test the hypothesis that an isolated increase in heart rate increases cardiac output. Evidence favoring this hypothesis is drawn from experimental studies in the fetal lamb. During the later stages of gestation, a spontaneous increase in heart rate increases cardiac output (7). Stroke volume and diastolic volume also increase with the increase in heart rate

Table 1. Heart rates during pacing of right sinus venosus (mean $\pm S E M, n=8$ )

\begin{tabular}{lc}
\hline & Heart rate $(\mathrm{bpm})$ \\
\hline Prepacing intrinsic & $156 \pm 7$ \\
Paced near intrinsic & $162 \pm 6$ \\
Paced at $125 \%$ of intrinsic & $190 \pm 7$ \\
Paced at $150 \%$ of intrinsic & $222 \pm 8$ \\
Postpacing intrinsic & $148 \pm 6$ \\
\hline
\end{tabular}

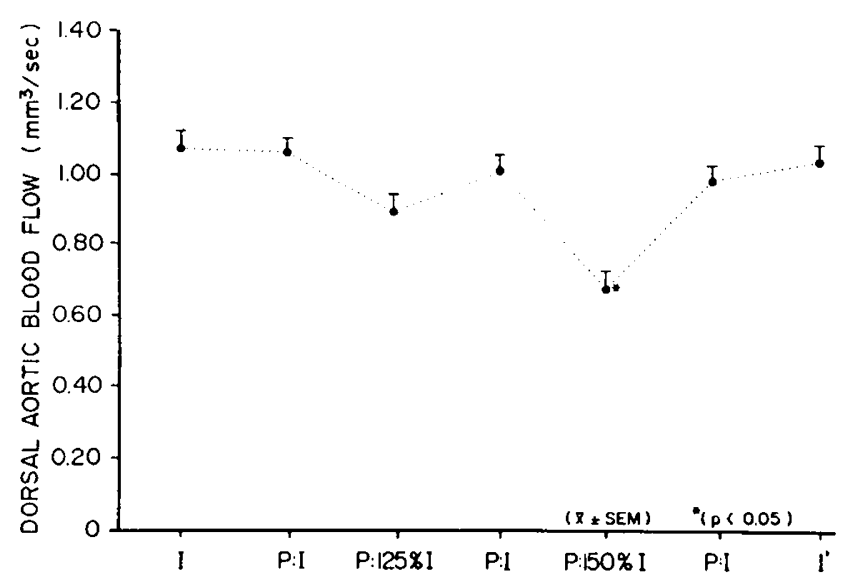

Fig. 1. Dorsal aortic blood flow during the sinus venosus pacing protocol. At intrinsic $(I)$, paced at intrinsic $(P: I)$, and postpacing intrinsic rates $\left(I^{\prime}\right)$ cardiac outputs were similar. However, dorsal aortic blood flow decreased with pacing at $125 \%$ of intrinsic heart rate $(P: 125 \% I)$ and at $150 \%$ of intrinsic heart rate $(P: 150 \% I)$.

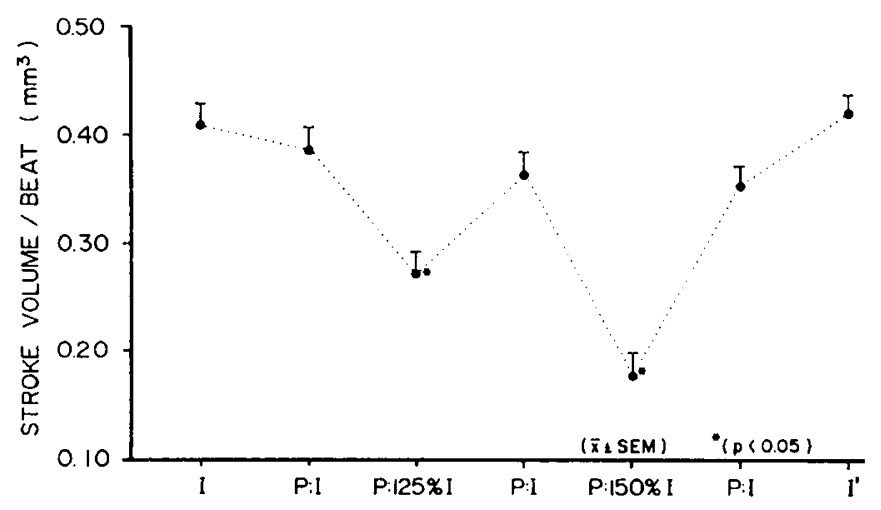

Fig. 2. Stroke volume per beat during the sinus venosus pacing protocol. Stroke volumes were similar at intrinsic $(I)$, paced intrinsic $(P: I)$, and postpacing intrinsic rates $\left(I^{\prime}\right)$. However, stroke volume decreased significantly with pacing at $125 \%$ of intrinsic heart rate $(P: I 25 \% I)$ and at $150 \%$ of intrinsic heart rate $(P: 150 \% I)$.

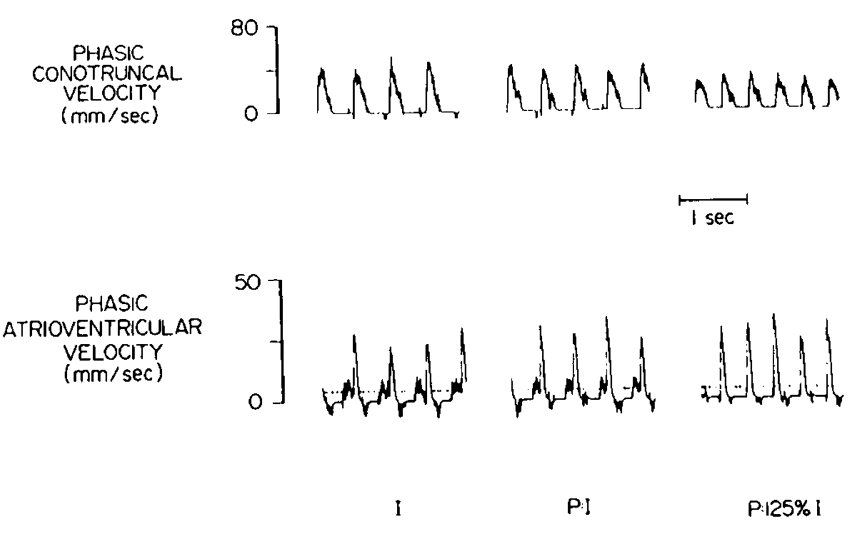

Fig. 3. Effect of sinus venosus pacing on phasic conotruncal outflow (upper panel) and phasic atrioventricular inflow (lower panel) blood velocity. At the intrinsic rate $(I)$, the low velocity passive filling wave preceded the active atrial wave. During pacing at the intrinsic rate $(P: I)$, the passive phase was somewhat shorter but phasic conotruncal velocity was maintained. During pacing at $125 \%$ of the intrinsic rate $(P: 125 \% I)$, the passive waveform was lost and the phasic conotruncal velocity decreased. No atrioventricular canal insufficiency was noted (negative waveform during ventricular systole) in the atrioventricular velocity profile. 

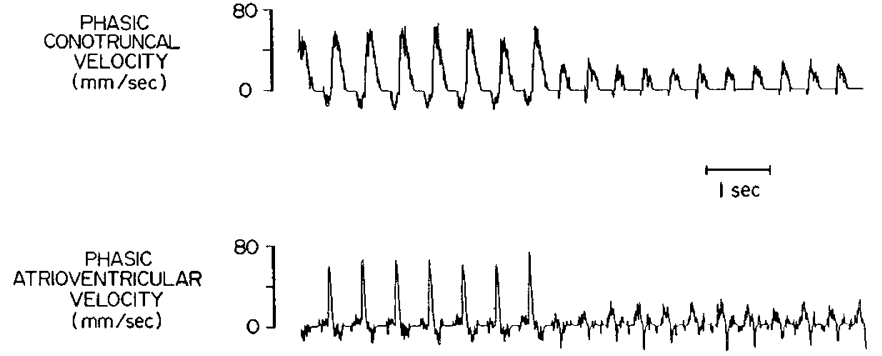

$$
1 \quad 1 \quad P: 1
$$

Fig. 4. Effect of ventricular pacing on conotruncal (upper panel) and atrioventricular (lower panel) blood flow velocity. Intrinsic cardiac contractions $(I)$ had the characteristic passive and active atrioventricular velocity profile. When pacing at the intrinsic rate $(P: I)$ (arrow), the atrioventricular velocity profiles flattened and the phasic conotruncal velocity decreased precipitously.

provided that the end-diastolic volume is maintained (8). In the late gestation fetal lamb, the relationship of heart rate and cardiac output is similar to the mature animal.

The early chick embryo did not respond like the late gestation fetal lamb to a spontaneous increase in heart rate. Rather, the chick embryo behaved more like the fetal lamb paced from the left atrium (8). We found that as heart rate increased above intrinsic, there was a marked compromise in cardiac performance. Measures of cardiac output and stroke volume decreased. Blood pressure and vascular resistance, may also have changed but these could not be measured because the pacing stimulus interfered with the servo-null pressure system.

The mechanism of this response is related at least in part to the passive phase of ventricular filling. At control rate, the atrioventricular canal blood velocity pattern in the chick was similar to the atrioventricular valve blood velocity profile recorded in the human fetus (9). With pacing at the intrinsic rate, the velocity profile showed a slight decrease in the low velocity, early diastolic signal that we interpreted as the passive phase of ventricular filling. As the pacing rate increased, the passive filling phase disappeared and the cardiac output decreased. Sinus venosus pacing may have also interfered with atrial contraction, and consequently, the active phase of ventricular filling.

Why the embryonic heart is critically dependent on passive filling is unclear. Optimum ventricular filling may be in part related to changes in ventricular morphology and consequently compliance. At stage 24 , the ventricular chamber is a trabecular network rather than a hollow cavity (10). With increasing heart rate, a decrease in chamber compliance may diminish ventricular filling. With cardiac pacing near intrinsic rate, active filling is likely maintained since the high velocity component appears unchanged.

Pacing the right sinus venosus presumably maintained the normal sequential pattern of myocardial excitation and contraction. During sinus venosus pacing near the intrinsic rate, cardiac output was maintained, stroke volume was normal, and the relationship of passive and active ventricular phase appeared similar to control. In addition, we did not find evidence of atrioventricular canal regurgitation, as there was no negative velocity noted on the atrioventricular velocity profiles from a sample volume that is greater than the canal dimensions.

Pacing the ventricle profoundly decreased antegrade blood flow from the ventricle to the conotruncus. However, again, we found no evidence of atrioventricular canal insufficiency. Thus, the mechanism for the drop in cardiac output was likely a loss of atrioventricular synchrony that compromised both active and passive ventricular filling.

The mechanisms that control the embryonic circulation are becoming more apparent. Isolated increase in heart rate is detrimental to the preinnervated embryo compared to the fetus and mature animal. The chick embryo may function at a heart rate that is optimum for cardiac output. Alterations in preload, however, may be more important for the regulation of hemodynamic function in the embryo. In the stage 24 chick, a $6 \%$ increase in circulating volume produces a $22 \%$ increase in stroke volume (11). Vascular resistance adjusts to cardiac output. With a decrease in cardiac output, resistance rises presumably to maintain blood flow to the embryo. With an increase in cardiac output, vascular resistance declines.

During morphogenesis cardiovascular control mechanisms emerge and change. In the preinnervated embryo, preload is likely the primary parameter for regulation of cardiac performance. With completion of morphogenesis and autonomic innervation, cardiovascular regulation becomes more complicated as a constellation of control mechanisms (heart rate, reflex-mediated changes in vascular resistance, and circulating catecholamines) regulate cardiovascular performance. Defining the relative contributions of these control mechanisms is essential to understanding normal development and the pathogenesis of abnormal regulation states such as essential hypertension.

\section{REFERENCES}

1. Nakazawa M, Clark EB, Hu N, Wispe J 1985 Effect of environmental hypothermia on vitelline artery blood pressure and vascular resistance in the stage 18, 21, and 24 chick embryo. Pediatr Res 19:651-654

2. Nakazawa M, Miyagawa S, Takao A, Clark EB, Hu N 1986 Hemodynamic effects of environmental hyperthermia in stage 18,21, and 24 chick embryos. Pediatr Res 20:1213-1215

3. Hamburger V, Hamilton HL 1951 A series of normal stages in the development of the chick embryo. J Morphol 88:49-92

4. Clark EB, Hu N 1982 Developmental hemodynamic changes in the chick embryo from stage 18 to 27 . Circ Res 51:810-815

5. Van Mierop LHS 1967 Location of pacemaker in chick embryo heart at the time of initiation of heart beat. Am J Physiol 212:407-415

6. Sperelakis N 1984 Developmental changes in membrane electrical properties of the heart. In: Sperelakis N (ed) Physiology and Pathophysiology of the Heart. Martinus-Nijhoff, Boston, pp 543-573

7. Rudolph AM, Heymann MA 1976 Cardiac output in the fetal lamb: the effects of spontaneous and induced changes of heart rate on right and left ventricular output. Am J Obstet Gynecol 124:183-192

8. Anderson PAW, Glick KL, Killam AP, Mainwaring RD 1986 The effect of heart rate on in utero left ventricular output in the fetal sheep. $J$ Physiol 372:557-573

9. Reed KL, Sahn DJ, Scagnelli S, Anderson CF, Shenker L 1986 Doppler echocardiographic studies of diastolic function in the human fetal heart: changes during gestation. JACC 8:391-395

10. Ben-Schachar G, Lucas RV, Arcilla R, Manasek FJ 1985 Muscular interventricular septum formation in the chick embryo. Circ Res 57:759-766

11. Wagman A, Clark EB 1985 Frank-Starling relationship in the developing cardiovascular system. In: Ferrens V, Rosenquist G, Weinstein C (eds) Cardiac Morphogenesis. Elsevier, New York, pp 245-252 